\title{
Erratum to: The effect of trastuzumab-based chemotherapy in small node-negative HER2-positive breast cancer
}

Mette S. van Ramshorst ${ }^{1}$ - Margriet van der Heiden-van der $\mathbf{L o o}^{2}$ •

Gwen M. H. E. Dackus ${ }^{1,3}$ - Sabine C. Linn ${ }^{1,3}$.

Gabe S. Sonke ${ }^{1}$

Published online: 17 August 2016

(c) Springer Science+Business Media New York 2016

\section{Erratum to: Breast Cancer Res Treat (2016) 158:361-371 \\ DOI 10.1007/s10549-016-3878-9}

In the original publication of the article, the $P$-value for two interactions tests was accidently swapped.

Under the heading Overall survival, the sentence starting with "Also, for HR status no...... 0 .95)" should read as "Also, for HR status no heterogeneity of treatment effect was found ( $P$-value for interaction 0.75$)$.
Under the heading Breast cancer-specific survival, the sentence starting with "Again, no evidence.... 0.75)" should read as "Again, no evidence for heterogeneity of treatment effect was found with tumor size $(P$-value for interaction 0.95).

In Table 1 of the Electronic Supplementary Material the hazard ratio for incidence year in the multivariate analysis was placed incorrectly by mistake. The corrected version of the table is given in supplementary material.

The conclusion remains unaffected by these corrections.

The online version of the original article can be found under doi:10.1007/s10549-016-3878-9.

Electronic supplementary material The online version of this article (doi:10.1007/s10549-016-3941-6) contains supplementary material, which is available to authorized users.

Gabe S. Sonke

g.sonke@nki.nl

1 Department of Medical Oncology, Netherlands Cancer Institute, Plesmanlaan 121, 1066CX Amsterdam, The Netherlands

2 Netherlands Comprehensive Cancer Organisation, Godebaldkwartier 419, 3511DT Utrecht, The Netherlands

3 Department of Molecular Pathology, Utrecht University Medical Center, Heidelberglaan 100, 3584CX Utrecht, The Netherlands 